\title{
Toll-like receptors and their role in experimental models of microbial infection
}

\author{
ST Qureshi ${ }^{1}$ and R Medzhitov ${ }^{1}$ \\ ${ }^{1}$ Section of Immunobiology, Yale University School of Medicine, New Haven, CT 06510, USA
}

\begin{abstract}
Effective host defense against microbial infection depends upon prompt recognition of pathogens, activation of immediate containment measures, and ultimately the generation of a specific and definitive adaptive immune response. The innate immune system of the host is responsible for providing constant surveillance against infection; when confronted by pathogens it deploys a series of rapidly acting antimicrobial effectors while simultaneously instructing the adaptive immune system as to the nature and context of the infectious threat. Pathogen recognition and activation of innate immunity is mediated by members of the Toll-like receptor (TLR) family through detection of conserved microbial structures that are absent from the host.

Experimental models of infection using TLR-deficient mice, as well as limited human studies, have clearly demonstrated the critical role of TLRs in host defense against most major groups of mammalian pathogens.
\end{abstract}

Genes and Immunity (2003) 4, 87-94. doi:10.1038/sj.gene.6363937

Keywords: toll-like receptor; innate immunity; infection

\section{Introduction}

Multicellular eukaryotic hosts are constantly challenged by microorganisms that are ubiquitous in the environment or present as colonizers, mainly of the epithelial surfaces. While this microbial presence may be beneficial under certain conditions, even a slight disruption of host-microbe homeostasis can have devastating and potentially fatal consequences. Survival of the host is therefore critically dependent on efficient mechanisms for containment of normal flora as well as rapid elimination of invading pathogens. Such defenses are present in many forms, including physical barriers (keratinized surfaces, tight epithelial junctions), nonspecific secretory products (gastric acid, bile, lysozyme), and immunologic defenses. The primary function of the immune system is to identify and eliminate infection, and this is achieved through two major elements; an adaptive component consisting of exquisitely specific $\mathrm{T}$ - and B-lymphocytes that undergo clonal selection and expansion upon presentation of a foreign antigen, as well as an innate component that is characterized by its rapid mobilization and activation of effector mechanisms upon microbial challenge. ${ }^{1}$ Of the two complementary subdivisions, the innate immune system has far more ancient evolutionary origins (being the only immune system of primitive multicellular organisms) and is transmitted via the germline without undergoing the somatic gene rearrangements that characterize antigen receptors of the adaptive immune system. Activation of innate immunity constitutes the first line of host defense

Correspondence: Dr ST Qureshi, Yale University School of Medicine, LCI 105, 333 Cedar Street, New Haven, CT 06520-8057, USA.

E-mail: salman.qureshi@yale.edu

Received 16 July 2002; revised 19 July 2002; accepted 23 July 2002 against infection, and provides instructive signals to the adaptive immune system by alerting it to the abnormal presence as well as the context of a microbial invader. ${ }^{2,3}$

The principal cellular components of the innate immune system include polymorphonuclear leukocytes (PMNs), tissue macrophages, dendritic cells (DCs), mast cells, and natural killer (NK) cells. These diverse lineages are all characterized by their ability to rapidly respond to infection or a breach of normal tissue architecture and to recognize and remove microbes and/or damaged host cells. DCs play an especially critical role in bridging innate to adaptive immunity through presentation of microbial breakdown products to T-lymphocytes in the context of the major histocompatibility complex (MHC), thereby initiating specific adaptive immune responses. A variety of effector mechanisms of the innate immune system have been extensively characterized, including exocytosis of neutrophil granule contents, phagolysosomal fusion in the host cell cytoplasm, and production of reactive oxygen species and nitrogen intermediates. Despite this detailed understanding, the mechanisms of pathogen recognition by cells of the innate immune system that precede the induction of such effectors have remained elusive. Recently, a major molecular mechanism for innate immune recognition of pathogens was established through identification of the mammalian Toll-like receptor (TLR) gene family.,

TLRs are one subset of a diverse group of molecules referred to as pattern recognition receptors (PRRs). ${ }^{1,3}$ Examples of previously identified PRRs include C-reactive protein, serum amyloid protein, and mannan-binding protein (all secreted into extracellular compartments), the mannose receptor and scavenger receptor (expressed on the macrophage cell surface), and the intracellular double-stranded RNA-activated protein kinase PKR. A major function of PRRs is to mobilize host 
defenses through several mechanisms including opsonization of foreign antigens and induction of host complement, coagulation, phagocytic, and proinflammatory signaling cascades. The microbial signatures recognized by PRRs have been termed pathogen-associated molecular patterns (PAMPs); these essential, invariant molecular structures are unique to microorganisms and shared among members of each class of infectious agents. To date, several of the 10 known members of the mammalian TLR family have clearly been shown to recognize these evolutionarily conserved microbial structures both in vitro, and in some cases in vivo, and to play an essential role in the maintenance of robust innate immunity. Identification of the TLR family represents a major advance in our understanding of host-pathogen interactions, and has stimulated tremendous interest in the field of innate immunity. In this article, we review selected aspects of TLR biology, and summarize studies that analyze the role of TLRs in experimental mouse models of infection. Brief reference will also be made, where possible, to work describing the role of TLRs in human host defense.

\section{Drosophila Toll and the identification of mammalian TLRs}

Identification of the first Toll receptor (and the origin of its nomenclature) has its origins in studies of embryonic development in Drosophila melanogaster. ${ }^{6}$ Through a mutagenesis screen, Drosophila Toll was identified as a gene controlling dorsoventral axis polarity in the developing fly embryo and determined to encode a transmembrane protein. ${ }^{7}$ Activation of Toll by its endogenous ligand, the cytokine-like peptide Spatzle, stimulates a signaling cascade culminating in the nuclear translocation of Dorsal, a transcription factor with homology to mammalian nuclear factor kappa-B (NFkB). This finding, combined with the recognition of the structural homology between the intracellular domains of Drosophila Toll and the mammalian interleukin-1 receptor (IL-1R) prompted investigation of the hypothesis that both molecules might also have functional similarities and play a role in innate immunity. Subsequent studies disclosed a critical role for Drosophila Toll in host defense; adult Toll mutant flies were unable to defend against experimental challenge with fungi and succumbed to overwhelming infection, yet retained normal responses to infection with Gram-negative bacteria. ${ }^{8}$ This susceptibility phenotype was shown to be due to a specific failure to synthesize the antifungal peptide Drosomycin in the Drosophila fat body (the equivalent of the mammalian liver) as a consequence of defective activation of dorsal immunity factor (DIF), another transactivator with homology to NFkB. ${ }^{9}$ Further investigations have shown that both fungal and Gram-positive infection activate the Toll pathway through the cleaved, activated form of Spatzle, and recruit members of an intracellular signaling cascade including the deathdomain-containing adaptor proteins dMyD88 and Tube, as well as the serine-threonine kinase Pelle, leading to degradation of the inhibitor protein Cactus and nuclear translocation of DIF. Notably, host defense against Gramnegative bacterial infection in Drosophila is mediated by a distinct recognition and signaling pathway defined by the Imd gene. ${ }^{10}$ The recently identified Imd gene product contains a death domain with closest sequence similarity to the mammalian tumor necrosis factor- $\alpha$ receptorinteracting protein (RIP). ${ }^{11}$ Imd regulates the expression of all antibacterial peptides, particularly those that are most active against Gram-negative bacteria, and in response to certain stimuli may also participate in the apoptotic response. Pathogen recognition and specificity of these proteolytic cascades occurs upstream of the most proximal members described for each pathway, Spatzle and Imd. Recognition of Gram-positive bacteria (but not fungi) and activation of Toll is mediated by a circulating peptidoglycan-recognition protein (PGRP-SA) encoded by the gene semmelweis. ${ }^{12}$ Another so-called PGRP (PGRP-LC) bearing a transmembrane domain was shown to mediate activation of the Drosophila Imd pathway in response to Gram-negative infection, indicating that members of the PGRP family represent critical microbial PRRs. ${ }^{13}$ Both soluble and membrane-bound mammalian PGRPs have been described, ${ }^{14}$ although their role in microbial host defense has not yet been defined. Based on structural homology, eight other Toll receptors have been identified in the Drosophila genome; all of these are expressed and presumably function during embryonic development, yet so far only the original member (Toll1) has been strongly implicated in host defense functions. ${ }^{15}$

\section{Mammalian TLRs}

Mammalian TLRs are a family of type 1 transmembrane receptors that share a common modular protein architecture consisting of a series of extracellular leucine-rich repeats flanked at the carboxy-terminal end by a cysteine-rich cluster that is connected by a single transmembrane span to a cytoplasmic Toll/Interleukin1 receptor (TIR) homology domain. LRRs are found among proteins with diverse functions, many of which do not have an obvious role in immune defense, and presumably mediate protein-protein and protein-ligand interactions. Among mammalian TLRs, the length of the extracellular LRR differs, and this variation may contribute to the specificity for various microbial ligands (although direct binding of PAMPs to TLRs has not been formally demonstrated to date). The intracellular TIR domain is essential for transmitting a downstream signal in response to TLR activation. The cytoplasmic domain of human TLR4, the first mammalian TLR identified, exhibits a higher degree of sequence conservation to its mouse counterpart compared to the corresponding extracellular domains, ${ }^{16}$ suggesting that greater constraints on structural variation exist for the signaling function of the receptor compared to its role in ligand recognition. All mammalian TLRs are known to signal through MyD88, a cytoplasmic adaptor protein that consists of an N-terminal death domain and C-terminal TIR domain. ${ }^{17}$ MyD88 is recruited to the TIR domain of activated TLRs and through a homophilic association mediates a signaling cascade that results in the activation and nuclear translocation of NFKB as well as activation of MAP kinases. ${ }^{18}$ Other recently identified adaptor proteins that interact with mammalian TLRs include TIR containing adaptor protein (TIRAP) ${ }^{19}$ that functions downstream of TLR4 in a MyD88-independent signaling pathway and Toll interacting protein (TOLLIP) ${ }^{20}$ that 
interacts with TLR2, TLR4, and IL-1R and has been shown to downregulate both IL-1 $\beta^{20}$ and TLR-mediated signaling in vitro. ${ }^{21,22}$

\section{Genetic mapping and regulation of mammalian TLR expression}

The chromosomal locations of the known members of the mammalian TLR family are shown in Table 1 . With the exception of human TLR10, all of the human TLRs identified to date have been cytogenetically localized. Three pairs of TLRs (TLR1/6, TLR2/3, TLR7/8) map to the same cytogenetic location, suggesting that they may have arisen via gene duplication events. The genomic location of several of the mouse TLR orthologues has not yet been published; however their chromosomal assignment may be predicted using comparative maps of the mouse and human genomes. The distribution of human TLR transcripts has been studied by real-time PCR analysis of normalized cDNA libraries and has revealed the most diverse expression pattern in spleen, peripheral blood leukocytes, lung, and gastrointestinal tract, consistent with the role of these tissues in immune defense and exposure to microbes. ${ }^{23}$ In the THP-1 leukemia cell line, several factors including cell differentiation, exposure to PAMPs, live bacteria and several different cytokines altered the levels of TLR mRNA. Incubation of whole human blood with Escherichia coli upregulated leukocyte expression of TLR3, TLR7, and MyD88 while TLR1, TLR6, and TLR9 expressions were downregulated. In this study, TLR8 and TLR10 were differentially regulated, with increased expression in monocytes and decreased levels in granulocytes. Analysis of the human TLR4 promoter has identified a functional interaction between the PU.1 and interferon consensus sequence binding protein (ICSBP) transcription factors that regulates the basal expression of this TLR family member in myeloid cells. ${ }^{24}$ The minimal promoter for human TLR2 has also been defined and contains binding sites for several transcription factors including Sp1 and Ets family members. ${ }^{25}$ Notably, the human and murine TLR2 promoters lack sequence homology and exhibit differential activation in response to several PAMPs including LPS and mycoplasma macrophage activating lipopeptide 2 kD (MALP 2).

Numerous studies, conducted mainly in vitro, have led to the identification of a large number of different ligands

Table 1 Chromosomal location of TLR family members

\begin{tabular}{lcc}
\hline Member & Human & Mouse \\
\hline TLR1 & $4 \mathrm{p} 14$ & Chr. $537.0 \mathrm{cM}$ \\
TLR2 & $4 \mathrm{q} 32$ & Chr. $3^{\mathrm{a}}$ \\
TLR3 & $4 \mathrm{q} 35$ & Chr. 8 \\
TLR4 & $9 \mathrm{q} 32-33$ & Chr. $433.0 \mathrm{cM}$ \\
TLR5 & $1 \mathrm{q} 41-42$ & Chr. $198.0 \mathrm{cM}$ \\
TLR6 & $4 \mathrm{p} 14$ & Chr. $537.0 \mathrm{cM}$ \\
TLR7 & Xp22.3 & Chr. X \\
TLR8 & Xp22 & Chr. X \\
TLR9 & $3 \mathrm{p} 21.3$ & Chr. $9^{\mathrm{a}}$ \\
TLR10 & $?$ & $?$ \\
\hline
\end{tabular}

${ }^{\text {a }}$ Denotes predicted location based on human-mouse comparative mapping data.
Table 2 Host defense defects conferred by defective TLR function

\begin{tabular}{lcc} 
Microbial class & Pathogen & Mouse mutation \\
\hline Gram-negative bacteria & Salmonella typhimurium & TLR4 \\
& Neisseria meningitidis & TLR4 \\
& Haemophilus influenza & TLR4 \\
Gram-positive bacteria & Staphylococcus aureus & TLR4, MyD88 \\
Fungus & Candida albicans & TLR4 \\
Parasite & Plasmodium berghei & MyD88 \\
& Toxoplasma gondii & MyD88 \\
Virus & Respiratory syncytial virus & TLR4 \\
Spirochete & Borrelia burgdorferi & TLR1, TLR2
\end{tabular}

for members of the TLR family. ${ }^{26}$ Recognition of a single ligand has been demonstrated for TLR3 (polyI:C, representative of viral double-stranded RNA ${ }^{27}$ ), TLR5 (bacterial flagellin $^{28}$ ), and TLR9 (hypomethylated CpG/ bacterial $\mathrm{DNA}^{29}$ ) while other TLRs exhibit broader specificities. TLR2 is capable of recognizing the widest repertoire of PAMPs including several associated with Gram-positive bacteria (peptidoglycan, lipoteichoic acid $^{30}$ ), mycobacteria (lipoarabinomannan, ${ }^{31}$ mycobacterial cell walls ${ }^{32}$ ), protozoan parasites (Trypanosoma cruzii GPI anchors, ${ }^{33}$ T. cruzii secreted protein Tc52 ${ }^{34}$ ), as well as microbial lipoproteins, ${ }^{35,36}$ glycoproteins, ${ }^{37}$ glycolipids, ${ }^{38}$ and nonenteric LPS. ${ }^{39}$ TLR6 associates with TLR2 to recognize several other PAMPs including the dipalmitoylated mycoplasma lipoprotein (MALP 2), ${ }^{40}$ phenol soluble modulin from Staphylococcus epidermidis, ${ }^{41}$ and fungal zymosan, ${ }^{42}$ while TLR1 associates with TLR2 to recognize triacylated lipoproteins such as Borrelia burgdorferi OspA. ${ }^{43}$ TLR4 recognizes LPS, ${ }^{44,45}$ the LPS mimetic drug Taxol, ${ }^{46}$ the fusion protein of respiratory syncytical virus (RSV), ${ }^{47}$ as well as fungal ligands. ${ }^{48,49}$ Recently, TLR7 and TLR8 were reported to recognize small imidazoquinoline compounds that have antiviral properties, $^{50,51}$ while a ligand for TLR10 has not yet been identified. Experimental confirmation of the role of the TLR family in host defense against microbes bearing one or more of these PAMPs has been obtained through the use of mice bearing spontaneous or genetically engineered defects of various TLR signaling components; these studies are summarized in the following section (see Table 2).

\section{Gram-negative bacteria}

Profound differences in susceptibility of certain inbred strains of mice to experimental challenge with Gramnegative bacteria were demonstrated long before the assignment of this phenotype to mutations of mammalian TLR4. Several studies using naturally mutant mice have implicated wild-type TLR4 in successful host defense against a variety of Gram-negative bacteria. The prototypic example is the $\mathrm{C} 3 \mathrm{H} / \mathrm{HeJ}$ mouse strain that is exquisitely sensitive to progressive, overwhelming infection by Salmonella typhimurium $\left(\mathrm{LD}_{50}<2\right.$ colony forming units (CFU)) compared to closely related strains $\left(\mathrm{LD}_{50}>2 \times 10^{3} \mathrm{CFU}\right) .^{52}$ This strain was also shown to be hyporesponsive to the proinflammatory properties of purified LPS, and both phenotypes were mapped to a single genetic locus on chromosome 4 initially termed 
Lps. ${ }^{53,54}$ Extensive studies led to the identification of TLR4 as the gene encoded by Lps, with $\mathrm{C} 3 \mathrm{H} / \mathrm{HeJ}$ mice harboring a spontaneous point mutation (Pro ${ }^{712}-\mathrm{His}^{712}$ ) in the cytoplasmic signaling domain. ${ }^{55,16}$ Two other mouse strains bear mutations of TLR4; C57BL10/ScN and its subline C57BL10/ScNCr carry a genomic deletion and do not express TLR4 while motor neuron degeneration (mnd) mutant mice have a large insertion within exon 2 that results in an aberrantly spliced transcript predicting a truncated protein. ${ }^{56}$ Both of these latter strains are hyporesponsive to LPS and therefore would also be predicted to exhibit profound susceptibility to overwhelming Gram-negative infection.

Relative to mice bearing a wild-type TLR4, C3H/HeJ and C57BL10/Sc mutant mice have also been shown to develop prolonged bacteremia after experimental infection with Neisseria meningitidis, another Gram-negative bacteria recognized for its exuberant production of LPS. ${ }^{57}$ Bacterial proliferation after intraperitoneal inoculation of $N$. meningitidis was several hundred times higher in TLR4 mutants compared to control mice of the same genetic background, and the median time to bacterial clearance from the blood was 6 days compared to $<1$ day for wild-type animals. Finally, in a recent study of respiratory tract infection due to the Gram-negative organism Haemophilus influenzae, TLR4 mutant mice exhibited reduced bacterial clearance from the lung associated with an impaired innate immune response characterized by reduced recruitment of neutrophils to the lung, impaired proinflammatory cytokine production in bronchoalveolar lavage (BAL), and reduced production of TNF- $\alpha$, MIP- $1 \alpha$ and activated NFKB in the airways. ${ }^{58}$ Survival or dissemination of infection from the respiratory tract infection was not reported in these studies.

Human hyporesponsiveness to inhaled endotoxin has been associated with a common, naturally occurring missense mutations (Asp299Gly and Thr399Ile) of the extracellular domain of TLR4. ${ }^{59}$ In this study, a strict individual correlation between the presence of this mutation and LPS hyporesponsiveness was not observed; however, comparison of groups bearing a wildtype vs mutant TLR4 demonstrated a significant reduction in airway responsiveness to progressively increasing doses of inhaled LPS. This phenotype was associated with reduced TLR4 protein expression on the airway epithelium. Transfection of wild-type TLR4 into airway epithelia or alveolar macrophages obtained from subjects bearing the missense mutation restored LPS responsiveness (as measured by IL- $1 \alpha$ production and TNF- $\alpha$ production, respectively). Although these data support the relevance of TLR4 to human LPS responsiveness, a retrospective analysis of the Asp299Gly mutation among 1047 patients in the United Kingdom failed to demonstrate an association of this mutation with the frequency or severity of documented meningococcal disease. ${ }^{60}$ This contrasts with the experimental data described for inbred mice bearing Tlr4 mutations and suggests that factors other than TLR4 are responsible for human host defense against meningococcal bacteremia. Most recently, a genotypic analysis of 91 patients admitted to French intensive-care units demonstrated a similar frequency of Asp299Gly or Thr399Ile TLR4 mutations compared to healthy blood donor controls, yet identified a significant association of the Asp299Gly mutation alone with septic shock. ${ }^{61}$

\section{Gram-positive bacteria}

A critical role for TLRs in host defense against Grampositive bacteria was strongly suggested by gene disruption experiments demonstrating that TLR2-deficient mice were unable to respond to peptidoglycan derived from these organisms. ${ }^{62}$ Confirmation of this phenotype was obtained by infecting TLR2-deficient mice with Staphylococcus aureus. ${ }^{63}$ In these studies, $T L R 2^{-/-}$mice were significantly more susceptible to lethal infection after a high-dose challenge $\left(1 \times 10^{7} \mathrm{CFU}\right)$ relative to wild-type controls and this was associated with significantly high bacterial counts in the blood and kidney (but not in the spleen). No significant differences in survival or bacterial proliferation in vivo were observed in wild-type or TLR2 $2^{-/-}$mice after a low-dose $\left(1 \times 10^{6} \mathrm{CFU}\right)$ challenge. Relative to $T L R 2^{-/-}$mice, $M y D 88^{-/-}$mice challenged with $1 \times 10^{7} \mathrm{CFU} S$. aureus died more rapidly and exhibited higher bacterial counts in the liver and kidney after both low- and high-dose challenge. Production of TNF- $\alpha$ and IL- 6 by peritoneal macrophages in response to heat-killed $S$. aureus was completely absent in $M y D 88^{-/-}$mice and only reduced in $T L R 2^{-/-}$macrophages, indicating a more definitive proinflammatory signaling defect conferred by MyD88 deficiency. The observed difference in host susceptibility between these two knockout mice suggests that additional TLRs or possibly other receptors that signal through $M y D 88$ are also involved in host defense against Gram-positive bacteria.

A study examining polymorphisms in TLR2 in a population of patients with septic shock has been published. ${ }^{64}$ A mutation (Arg753Gln) within a group of highly conserved amino acids at the C-terminus of TLR2 was identified in $3 \%$ of healthy blood donor controls; transfection of this mutant into human epithelial kidney (HEK 293T) cells revealed decreased cellular activation in response to bacterial lipoproteins but not LPS. The Arg753Gln TLR2 variant was identified in two of 91 patients with septic shock; while this association was not statistically significant, both patients bearing the polymorphism were infected with S. aureus, suggesting that it may be a predisposing factor for severe Gram-positive infection.

\section{Fungal infection}

As described above, adult Drosophila Toll mutants were first shown to be highly susceptible to overwhelming fungal infection. In the light of this observation, it is somewhat surprising that there is relatively little evidence to implicate mammalian TLRs in antifungal host defense. In vitro, TLR2 has been shown to recognize zymosan, a yeast cell wall particle, and TLR4 plays a role in the induction of cytokines and intracellular signaling in response to stimulation with Aspergillus fumigatus and Cryptococcal polysaccharide capsule, respectively. A recent study compared the host response of $\mathrm{C} 3 \mathrm{H} / \mathrm{HeJ}$ mice to the genetically similar $\mathrm{C} 3 \mathrm{H} / \mathrm{HeN}$ (wild-type TLR4) strain after a nonlethal systemic challenge with the opportunistic fungus Candida albicans. At both day 1 and day 7 after experimental challenge, the $\mathrm{C} 3 \mathrm{H} / \mathrm{HeJ}$ strain was found to have a significantly (10-fold) higher fungal load in the kidneys compared to $\mathrm{C} 3 \mathrm{H} / \mathrm{HeN}$ control mice; however, later time points evaluating the clearance or progression of infection and its impact on mortality were 
not reported. It may be concluded from this study that TLR4 plays a role in restricting fungal replication in certain organs, although whether this ultimately translates into a deleterious outcome is unclear. The mechanism of impaired host defense in $\mathrm{C} 3 \mathrm{H} / \mathrm{HeJ}$ mice during Candidal infection appears to be reduced synthesis of the chemokines KC and MIP-2 by macrophages, resulting in reduced neutrophil recruitment. Phagocytosis and killing of C. albicans by neutrophils was similar between $\mathrm{C} 3 \mathrm{H} /$ $\mathrm{HeJ}$ and $\mathrm{C} 3 \mathrm{H} / \mathrm{HeN}$ strains, and no difference in proinflammatory cytokine production (TNF- $\alpha, \mathrm{IL}-1 \alpha$, IL-1 $\beta$ ) was observed.

\section{Parasitic infection}

Participation of TLRs in host defense against experimental malaria was studied using $M y D 88^{-/-}$mice infected with a virulent strain of Plasmodium berghei. ${ }^{65}$ In this experimental model, lymphocyte-mediated liver injury is dependent on an elevated serum level of IL-12 and independent of IL-18. Interestingly, endogenous or exogenous IL-12 is essential for clearance of attenuated strains of malaria. MyD88 $8^{-/-}$mice infected with $P$. berghei showed similar mortality and parasitemia compared to wild-type controls: however they were resistant to liver injury and had significantly lower levels of serum IL-12, implicating the TLR pathway in this aspect of the host response. Infection of $\mathrm{C} 3 \mathrm{H} / \mathrm{HeJ}$ (TLR4-mutant) mice, TLR6-deficient or TLR2/TLR4 doubly deficient mice did not reveal significant reductions in IL-12, suggesting that other TLR family member(s) may be responsible for mediating malarial recognition, or alternatively that a TLR-independent, MyD88-dependent signaling pathway is activated by $P$. berghei. The genetic control of host defense against experimental malaria is complex; independent studies using Plasmodium chabaudii have identified quantitative trait loci (QTLs) conferring host susceptibility on mouse chromosomes 9 (Char1), 8 (Char2), 17 (Char3) and 3 (Char4). ${ }^{66}$ Through in vivo studies, MyD88 has been shown to play a critical role in resistance to Toxoplasma gondii, an apicomplexan parasite. ${ }^{67}$ In this study, MyD88/- mice failed to control acute T. gondii infection, succumbing 13-16 days after intraperitoneal parasite inoculation, while control mice survived for $>60$ days; a similar pattern of early mortality was observed for IL-12p40-deficient mice. This susceptibility phenotype was associated with a dramatically increased parasite burden in both knockout strains, indicating that both IL12 and MyD88 are essential for efficient control of T. gondii replication by the host. $M y D 88^{-/-}$mice exhibited a defect in parasite-induced IL-12-dependent IFN- $\gamma$ production in vivo, a pathway that was previously shown to mediate host resistance to $T$. gondii. In vitro stimulation of bonemarrow-derived neutrophils, splenic DC, and thioglycollate-elicited peritoneal macrophages from these knockout mice with soluble tachyzoite antigen (STAg) or live tachyzoites confirmed that parasite-induced IL-12 production is dependent on $M y D 88$, although no TLR candidates (including TLR2 and TLR4) have yet been formally implicated in this pathway.

\section{Viral infection}

Evidence for the participation of TLRs in host defense against viral infection was reported using RSV infection of TLR4-deficient C57BL/10ScNCr mice. ${ }^{68}$ These studies were based on earlier findings that implicated TLR 4 and CD14 in the recognition of RSV fusion (F) protein. ${ }^{47}$ Secretion of IL-6, IL-8, TNF- $\alpha$, and IL- $1 \beta$ was induced by incubation of human monocytes or thioglycollate-induced mouse peritoneal macrophages with purified RSV F protein or UV-inactivated whole RSV virus. In contrast, macrophages from mice deficient in either CD14 or TLR4 did not secrete IL-6 when incubated with RSV F protein. Following intranasal infection of TLR4-deficient mice with infectious RSV, delayed clearance was observed, with 100- to 1000-fold higher viral titers in the lungs of the $\mathrm{C} 57 \mathrm{BL} / 10 \mathrm{ScNCr}$ strain relative to control mice. Characterization of the immune response following RSV infection revealed decreased pulmonary infiltration by NK cells and CD14+ cells, diminished IL-12 expression by BAL cells, and impaired perforin-dependent cytotoxicity of BAL NK cells. These differences in innate immune response were not observed in parallel studies using a mouse-adapted influenza virus, indicating that TLR4-mediated viral recognition and host defense may be specific to RSV. It has been suggested that the RSV F protein may have specifically evolved to recognize and exploit TLR4, whose primary function is LPS recognition. Evidence for viral strategies to evade recognition by TLRs has also been derived from sequence analysis of two genes of the vaccinia virus, A46R and A52R, that have sequence similarity to TIR domains of mammalian TLRs. ${ }^{69}$ Through in vitro studies, these gene products were shown to inhibit IL-1 and TLR4-mediated NFKB activation, suggesting that they may function as antagonists of the innate immune response during intracellular viral replication. TLR3 is another recently identified candidate for viral recognition and host defense, arising from studies using poly(I:C), a synthetic double-stranded RNA (dsRNA) analogue that is a molecular pattern associated with viral infection. ${ }^{27}$ Cell transfection studies with dsRNA triggered $\mathrm{NF \kappa B}$ activation and production of type I interferons that are known for their antiviral properties. Macrophages from mice deficient in TLR3 exhibited specific defects in the secretion of IL- 6 and IL12 , activation of $\mathrm{NF \kappa} B$, and induction of IFN- $\alpha$ or IFN- $\beta$, in response to poly(I:C), while B-cells from these mice did not upregulate surface expression of CD69, CD80, or CD86 in response to incubation with poly(I:C) or viral genomic dsRNA. TLR3-deficient mice were resistant to poly(I:C)-induced shock associated with reduced serum levels of IL-12. Although live virus infection of these mice has not yet been reported, based on the current data it is likely that TLR3 plays a crucial role in antiviral host defense. Recent reports also suggest that mouse TLR7 and human TLR7 and TLR8 may participate in antiviral host defense, based on the observation that small antiviral imidazoquinoline compounds are capable of stimulating cellular activation through these receptors. ${ }^{50,51}$

\section{Spirochetal infection}

Innate host defense against the spirochete $B$. burgdorferi has been studied using TLR1- and TLR2-deficient mice. ${ }^{43,70}$ While initial in vitro studies showed that cellular recognition of lipoproteins from a variety of microorganisms including Mycobacteria, Mycoplasma sp. and the spirochetes Treponema pallidum and B. burgdorferi 
is mediated by TLR2, ${ }^{35,36,71}$ more recent work has revealed a functional interaction between TLR2 and TLR1 that enhances cellular signaling in response to $B$. burgdorferi lipoprotein OspA. ${ }^{43}$ Macrophages from both TLR1- or TLR2-deficient mice incubated with OspA exhibit impaired cytokine and nitric oxide (NO) production compared to wild-type controls. Sonicated preparations of whole $B$. burgdorferi stimulate IL-6 or NO production by $T L R 2^{-/-}$macrophages, but only at 10 100-fold higher concentrations than that required for wild-type cells, suggesting that alternative (though less potent) TLR2-independent stimulatory pathways exist. Infection of $T L R 1^{-/-}$and TLR2 $2^{-/-}$mice with B. burgdorferi produced a mild arthritis and carditis at 2 weeks postinfection with disease resolution by 8 weeks, a pattern that was similar to wild-type controls. Quantification of spirochetal load in various tissues (skin, ankle joints, ears, and hearts) at 2 weeks post-infection using PCR demonstrated elevated numbers of spirochetes at all sites in $T L R 1^{-/-}$and $T L R 2^{-/-}$mice compared to wild-type controls. In one study, $T L R 2^{-/-}$mice failed to clear these high concentrations of spirochetes from their tissues at 4 weeks after infection, harboring 100-fold more bacteria than littermate controls in the ears and 10-fold more bacteria in the heart at both time points compared to wild-type or heterozygous controls. ${ }^{70}$ These observations convincingly demonstrate a critical role for both TLR1 and TLR2 in host defense during the first several weeks following infection with $B$. burgdorferi. In contrast, neither TLR1 nor TLR2 deficiency markedly influenced the overall course of disease or the acquired humoral immune response as determined by ELISA and immunoblotting, suggesting that the presence of either genetic defect did not prevent the generation of an effective adaptive immune response associated with preserved Blymphocyte function. Selective humoral hyporesponsiveness to vaccination with recombinant OspA was demonstrated in a small proportion of healthy human volunteers. Impaired production of TNF- $\alpha$ and IL- 6 was observed following ex vivo stimulation of macrophages from these individuals with lipidated OspA. No significant mutations of either the TLR1 or TLR2 gene were detected in the low responders. Flow cytometric analysis of peripheral blood mononuclear cells from each of these individuals did reveal markedly reduced cell surface expression of TLR1 (but not TLR2), suggesting that their hyporesponsive phenotype is due to altered TLR1 function.

\section{Mycobacterial infection}

Experimental models specifically analyzing the contribution of TLRs to host defense against live mycobacteria have not yet been published. Nevertheless, extensive in vitro data implicating TLR2 in the recognition of mycobacterial lipoarabinomannan, ${ }^{31}$ a soluble tuberculosis factor, ${ }^{22}$ whole Mycobacterium avium, ${ }^{71}$ and Mycobacterium tuberculosis ${ }^{72,73}$ strongly suggest that one or more TLRs participate in mycobacterial host defense. Published genetic analyses of experimental mycobacterial infection among different strains of inbred mice have identified significant QTL for tuberculosis susceptibility (sst1) on mouse chromosome $1^{74}$ as well as resistance (Trl1-3) on mouse chromosomes 1, 3, and $7 .^{75}$ TLR5 is located on chromosome 1 but is unlikely to be a candidate gene for either the susceptibility or resistance phenotypes since it maps distal to both candidate intervals. Alternatively, TLR2 may be a potential candidate locus for resistance to infection by $M$. tuberculosis since it has been localized to chromosome 3 (though not precisely mapped).

Sequence analysis of the intracellular domain of TLR2 was performed in a group of 86 human subjects infected with Mycobacterium leprae, the causative agent of leprosy, in Korea. ${ }^{76}$ In this study, patients were categorized as having one of the two major disease patterns, tuberculoid (characterized by few bacteria and a pronounced cell-mediated host response) and lepromatous (characterized by a large bacillary load and relatively weak host immune response). Ten of $45(22 \%)$ of the lepromatous patients exhibited a C-T transition at nucleotide 2029, resulting in replacement of a conserved arginine residue with tryptophan at amino acid 677, while none of the tuberculoid patients nor control group carried this mutation. Functional studies were not performed in these patients; however, the authors postulate that this TLR2 mutation might result in defective mycobacterial phagocytosis by macrophages, resulting in an impaired Th1 response characteristic of patients with lepromatous leprosy.

\section{Future directions}

Although tremendous progress has been made towards understanding the biology of the TLRs and their essential role in innate immune recognition, many areas remain to be explored. Among the most vexing issues is the mechanism by which TLRs specifically recognize their ligands. In the absence of definitive experimental data, several alternative models have been proposed, including direct physical contact between the microbial ligand and TLR, ${ }^{77}$ formation of a macromolecular complex including ligand, adaptor molecule(s) and TLR(s), or generation of endogenous ligands in response to infection (analogous to the system in Drosophila) that bind and activate TLRs. Recognition of endogenous ligands by TLRs is also an area of active investigation due to its potential relevance to autoimmune diseases, noninfectious inflammatory disorders, and elimination of neoplastic or damaged cells. A number of putative endogenous TLR ligands have already been studied in vitro, including heat shock protein 60 (Hsp60), ${ }^{78}$ fibronectin, ${ }^{79}$ and the extra domain A of fibrinogen. ${ }^{80}$ Recent data have also demonstrated that low-molecular weight soluble hyaluronan (sHA) fragments derived from the extracellular matrix at sites of inflammation are able to induce DC maturation in vitro as well as in vivo through TLR4. ${ }^{81}$ Finally, identification of all relevant TLR ligands, combined with a comprehensive understanding of the different intracellular signaling pathways and repertoire of downstream targets activated by individual TLRs and their various combinations will significantly advance our understanding of host-pathogen interactions.

\section{Acknowledgements}

The authors thank Markus Schnare for critically reviewing the manuscript. 


\section{References}

1 Medzhitov R, Janeway CA Jr. Innate immunity: the virtues of a nonclonal system of recognition. Cell 1997; 91: 295-298.

2 Schnare $\mathrm{M}$ et al. Toll-like receptors control activation of adaptive immune responses. Nat Immunol 2001; 2: 947-950.

3 Janeway CA Jr, Medzhitov R. Innate immune recognition. Annu Rev Immunol 2002; 20: 197-216.

4 Medzhitov R, Preston-Hurlburt P, Janeway CA Jr. A human homologue of the Drosophila Toll protein signals activation of adaptive immunity. Nature 1997; 388: 394-397.

5 Rock FL et al. A family of human receptors structurally related to Drosophila Toll. Proc Natl Acad Sci USA 1998; 95: 588-593.

6 Belvin MP, Anderson KV. A conserved signaling pathway: the Drosophila toll-dorsal pathway. Annu Rev Cell Dev Biol 1996; 12: 393-416.

7 Hashimoto C, Hudson KL, Anderson KV. The Toll gene of Drosophila, required for dorsal-ventral embryonic polarity, appears to encode a transmembrane protein. Cell 1988; 52 269-279.

8 Lemaitre B et al. The dorsoventral regulatory gene cassette spatzle/Toll/cactus controls the potent antifungal response in Drosophila adults. Cell 1996; 86: 973-983.

9 Meng X, Khanuja BS, Ip YT. Toll receptor-mediated Drosophila immune response requires Dif, an NF-kappaB factor. Genes Dev 1999; 13: 792-797.

10 Lemaitre $\mathrm{B}$ et al. A recessive mutation, immune deficiency (imd), defines two distinct control pathways in the Drosophila host defense. Proc Natl Acad Sci U S A 1995; 92: 9465-9469.

11 Georgel $P$ et al. Drosophila immune deficiency (IMD) is a death domain protein that activates antibacterial defense and can promote apoptosis. Dev Cell 2001; 1: 503-514.

12 Michel T et al. Drosophila Toll is activated by Gram-positive bacteria through a circulating peptidoglycan recognition protein. Nature 2001; 414: 756-759.

13 Gottar $\mathrm{M}$ et al. The Drosophila immune response against Gramnegative bacteria is mediated by a peptidoglycan recognition protein. Nature 2002; 416: 640-644.

14 Liu C et al. Peptidoglycan recognition proteins: a novel family of four human innate immunity pattern recognition molecules. J Biol Chem 2001; 276: 34686-34694.

15 Hoffmann JA, Reichhart JM. Drosophila innate immunity: an evolutionary perspective. Nat Immunol 2002; 3: 121-126.

16 Qureshi ST et al. Endotoxin-tolerant mice have mutations in Toll-like receptor 4 (Tlr4). J Exp Med 1999; 189: 615-625.

17 Medzhitov R et al. MyD88 is an adaptor protein in the hToll/ IL-1 receptor family signaling pathways. Mol Cell 1998; 2: 253-258.

18 Medzhitov R. Toll-like receptors and innate immunity. Nat Rev Immunol 2001; 1: 135-145.

19 Horng T, Barton GM, Medzhitov R. TIRAP: an adapter molecule in the Toll signaling pathway. Nat Immunol 2001; 2 : 835-841.

20 Burns K et al. Tollip, a new component of the IL-1RI pathway, links IRAK to the IL-1 receptor. Nat Cell Biol 2000; 2: 346-351.

21 Zhang G, Ghosh S. Negative regulation of toll-like receptormediated signaling by Tollip. J Biol Chem 2002; 277: 7059-7065.

22 Bulut $\mathrm{Y}$ et al. Cooperation of Toll-like receptor 2 and 6 for cellular activation by soluble tuberculosis factor and Borrelia burgdorferi outer surface protein A lipoprotein: role of Tollinteracting protein and IL-1 receptor signaling molecules in Toll-like receptor 2 signaling. J Immunol 2001; 167: 987-994.

23 Zarember KA, Godowski PJ. Tissue expression of human Tolllike receptors and differential regulation of Toll-like receptor mRNAs in leukocytes in response to microbes, their products, and cytokines. I Immunol 2002; 168: 554-561.

24 Rehli $\mathrm{M}$ et al. PU.1 and interferon consensus sequence-binding protein regulate the myeloid expression of the human Toll-like receptor 4 gene. J Biol Chem 2000; 275: 9773-9781.
25 Haehnel V et al. Transcriptional regulation of the human tolllike receptor 2 gene in monocytes and macrophages. I Immunol 2002; 168: 5629-5637.

26 Lien E, Ingalls RR. Toll-like receptors. Crit Care Med 2002; 30(Suppl): S1-S11.

27 Alexopoulou L et al. Recognition of double-stranded RNA and activation of NF-kappaB by Toll-like receptor 3. Nature 2001; 413: 732-738.

28 Hayashi $\mathrm{F}$ et al. The innate immune response to bacterial flagellin is mediated by Toll-like receptor 5. Nature 2001; 410: 1099-1103.

$29 \mathrm{Hemmi} \mathrm{H}$ et al. A Toll-like receptor recognizes bacterial DNA. Nature 2000; 408: 740-755.

30 Schwandner $\mathrm{R}$ et al. Peptidoglycan- and lipoteichoic acidinduced cell activation is mediated by toll-like receptor 2 . J Biol Chem 1999; 274: 17406-17409.

31 Means TK et al. The CD14 ligands lipoarabinomannan and lipopolysaccharide differ in their requirement for Toll-like receptors. I Immunol 1999; 163: 6748-6755.

32 Tsuji $\mathrm{S}$ et al. Maturation of human dendritic cells by cell wall skeleton of Mycobacterium bovis bacillus Calmette-Guerin: involvement of toll-like receptors. Infect Immun 2000; 68: 6883-6890.

33 Campos MA et al. Activation of Toll-like receptor-2 by glycosylphosphatidylinositol anchors from a protozoan parasite. J Immunol 2001; 167: 416-423.

34 Ouaissi A et al. The Trypanosoma cruzi Tc52-released protein induces human dendritic cell maturation, signals via Toll-like receptor 2, and confers Protection Against Lethal Infection. J Immunol 2002; 168: 6366-6374.

35 Aliprantis $\mathrm{AO}$ et al. Cell activation and apoptosis by bacterial lipoproteins through toll-like receptor-2. Science 1999; 285: 736-739.

36 Brightbill HD et al. Host defense mechanisms triggered by microbial lipoproteins through toll-like receptors. Science 1999; 285: 732-736.

37 Sugawara $\mathrm{S}$ et al. Monocytic cell activation by nonendotoxic glycoprotein from Prevotella intermedia ATCC 25611 is mediated by toll-like receptor 2. Infect Immun 2001; 69: 4951-4957.

38 Opitz B et al. Toll-like receptor-2 mediates treponema glycolipid and lipoteichoic acid-induced NF-kappaB translocation. J Biol Chem 2001; 276: 22041-22047.

39 Werts C et al. Leptospiral lipopolysaccharide activates cells through a TLR2-dependent mechanism. Nat Immunol 2001; 2: 346-352.

40 Takeuchi $\mathrm{O}$ et al. Discrimination of bacterial lipoproteins by Toll-like receptor 6. Int Immunol 2001; 13: 933-940.

41 Hajjar AM et al. Cutting edge: functional interactions between toll-like receptor (TLR) 2 and TLR1 or TLR6 in response to phenol-soluble modulin. I Immunol 2001; 166: 15-19.

42 Ozinsky A et al. The repertoire for pattern recognition of pathogens by the innate immune system is defined by cooperation between toll-like receptors. Proc Natl Acad Sci USA 2000; 97: 13766-13771.

43 Alexopoulou $\mathrm{L}$ et al. Hyporesponsiveness to vaccination with Borrelia burgdorferi OspA in humans and in TLR1- and TLR2deficient mice. Nat Med 2002; 8: 878-884.

44 Chow JC et al. Toll-like receptor- 4 mediates lipopolysaccharide-induced signal transduction. J Biol Chem 1999; 274: 10689-10692.

45 Hirschfeld $\mathrm{M}$ et al. Cutting edge: repurification of lipopolysaccharide eliminates signaling through both human and murine toll-like receptor 2. J Immunol 2000; 165: 618-622.

46 Kawasaki $\mathrm{K}$ et al. Mouse toll-like receptor 4.MD-2 complex mediates lipopolysaccharide-mimetic signal transduction by Taxol. J Biol Chem 2000; 275: 2251-2254.

47 Kurt-Jones EA et al. Pattern recognition receptors TLR4 and CD14 mediate response to respiratory syncytial virus. Nat Immunol 2000; 1: 398-401. 
48 Shoham $\mathrm{S}$ et al. Toll-like receptor 4 mediates intracellular signaling without TNF-alpha release in response to Cryptococcus neoformans polysaccharide capsule. J Immunol 2001; 166: 4620-4626.

49 Wang JE et al. Involvement of CD14 and toll-like receptors in activation of human monocytes by Aspergillus fumigatus hyphae. Infect Immun 2001; 69: 2402-2406.

$50 \mathrm{Hemmi} \mathrm{H}$ et al. Small anti-viral compounds activate immune cells via the TLR7 MyD88-dependent signaling pathway. Nat Immunol 2002; 3: 196-200.

51 Jurk $M$ et al. Human TLR7 or TLR8 independently confer responsiveness to the antiviral compound R-848. Nat Immunol 2002; 3: 499.

52 O'Brien AD et al. Genetic control of susceptibility to Salmonella typhimurium in mice: role of the LPS gene. J Immunol 1980; 124: 20-24.

53 Watson J, Riblet R, Taylor BA. The response of recombinant inbred strains of mice to bacterial lipopolysaccharides. I Immunol 1977; 118: 2088-2093.

54 Watson $\mathrm{J}$ et al. The genetic mapping of a defective LPS response gene in $\mathrm{C} 3 \mathrm{H} / \mathrm{HeJ}$ mice. I Immunol 1978; 120: 422-424.

55 Poltorak A et al. Defective LPS signaling in $\mathrm{C} 3 \mathrm{H} / \mathrm{HeJ}$ and C57BL/10ScCr mice: mutations in Tlr4 gene. Science 1998; 282: 2085-2088.

$56 \mathrm{Bihl} \mathrm{F}$ et al. LPS-hyporesponsiveness of mnd mice is associated with a mutation in Toll-like receptor 4. Genes Immun 2001; 2: 56-59.

57 Woods JP et al. Mouse genetic locus Lps influences susceptibility to Neisseria meningitidis infection. Infect Immun 1988; 56 : 1950-1955.

58 Wang $X$ et al. Toll-like receptor 4 mediates innate immune responses to Haemophilus influenzae infection in mouse lung. I Immunol 2002; 168: 810-815.

59 Arbour NC et al. TLR4 mutations are associated with endotoxin hyporesponsiveness in humans. Nat Genet 2000; 25: 187-191.

60 Read RC et al. A functional polymorphism of toll-like receptor 4 is not associated with likelihood or severity of meningococcal disease. I Infect Dis 2001; 184: 640-642.

61 Lorenz E et al. Relevance of mutations in the TLR4 receptor in patients with Gram-negative septic shock. Arch Intern Med 2002; 162: 1028-1032.

62 Takeuchi $\mathrm{O}$ et al. Differential roles of TLR2 and TLR4 in recognition of Gram-negative and Gram-positive bacterial cell wall components. Immunity 1999; 11: 443-451.

63 Takeuchi O, Hoshino K, Akira S. Cutting edge: TLR2-deficient and MyD88-deficient mice are highly susceptible to Staphylococcus aureus infection. J Immunol 2000; 165: 5392-5396.

64 Lorenz E et al. A novel polymorphism in the toll-like receptor 2 gene and its potential association with Staphylococcal infection. Infect Immun 2000; 68: 6398-6401.
65 Adachi K et al. Plasmodium berghei infection in mice induces liver injury by an IL-12- and toll-like receptor/myeloid differentiation factor 88-dependent mechanism. J Immunol 2001; 167: 5928-5934.

66 Fortin A, Stevenson MM, Gros P. Complex genetic control of susceptibility to malaria in mice. Genes Immun 2002; 3: 177-186.

67 Scanga CA et al. Cutting edge: MyD88 is required for resistance to Toxoplasma gondii infection and regulates parasite-induced IL-12 production by dendritic cells. J Immunol 2002; 168: 5997-6001.

68 Haynes LM et al. Involvement of toll-like receptor 4 in innate immunity to respiratory syncytial virus. J Virol 2001; 75: 10730-10737.

69 Bowie A et al. A46R and A52R from vaccinia virus are antagonists of host IL-1 and toll-like receptor signaling. Proc Natl Acad Sci USA 2000; 97: 10162-10167.

70 Wooten RM et al. Toll-like receptor 2 is required for innate, but not acquired, host defense to Borrelia burgdorferi. I Immunol 2002; 168: 348-355.

71 Lien E et al. Toll-like receptor 2 functions as a pattern recognition receptor for diverse bacterial products. J Biol Chem 1999; 274: 33419-33425.

72 Means TK et al. Human toll-like receptors mediate cellular activation by Mycobacterium tuberculosis. J Immunol 1999; 163: 3920-3927.

73 Underhill DM et al. Toll-like receptor-2 mediates mycobacteria-induced proinflammatory signaling in macrophages. Proc Natl Acad Sci USA 1999; 96: 14459-14463.

74 Kramnik I et al. Genetic control of resistance to experimental infection with virulent Mycobacterium tuberculosis. Proc Natl Acad Sci U S A 2000; 97: 8560-8565.

75 Mitsos LM et al. Genetic control of susceptibility to infection with Mycobacterium tuberculosis in mice. Genes Immun 2000; 1 467-477.

76 Kang TJ, Chae GT. Detection of Toll-like receptor 2 (TLR2) mutation in the lepromatous leprosy patients. FEMS Immunol Med Microbiol 2001; 31: 53-58.

77 Poltorak A et al. Physical contact between lipopolysaccharide and toll-like receptor 4 revealed by genetic complementation. Proc Natl Acad Sci USA 2000; 97: 2163-2167.

78 Ohashi $\mathrm{K}$ et al. Cutting edge: heat shock protein 60 is a putative endogenous ligand of the toll-like receptor- 4 complex. J Immunol 2000; 164: 558-561.

79 Okamura Y et al. The extra domain A of fibronectin activates Toll-like receptor 4. J Biol Chem 2001; 276: 10229-10233.

80 Smiley ST, King JA, Hancock WW. Fibrinogen stimulates macrophage chemokine secretion through toll-like receptor 4 . Immunol 2001; 167: 2887-2894.

81 Termeer $\mathrm{C}$ et al. Oligosaccharides of Hyaluronan activate dendritic cells via toll-like receptor 4. J Exp Med 2002; 195 99-111. 